\title{
Applying models of spatial interpolation for the approximation of responses in the refinement of parameters of finite element models
}

\author{
Anatoly Pikhalov ${ }^{1}$, and Anton Zabelin ${ }^{2, *}$ \\ ${ }^{1}$ Irkutsk National Research Technical University, 664074, Lermontova str., 83, Irkutsk, Russia \\ ${ }^{2}$ Irkutsk State University of Communications, 664074, Chernyshevsky str., 15, Irkutsk, Russia
}

\begin{abstract}
The numerical experiment on refining the parameters of the finite element model of the beam by the method of approximating the responses is presented in the article. As mathematical models of joint-stock companies are used: linear combinations of radial-basis functions, and Kriging-models. These models are generated in the work on the basis of Latin squares and depend on the parameters to be refined (the moduli of elasticity of finite element groups of the beam). To obtain optimal values of the parameters, a genetic optimization method was used. The results of solving the optimization problem showed a high level of coincidence of the parameter values with a combination of response models obtained from dynamic and static types of calculations. It was also shown that when solving the problems of finite element models, it is sufficient to use models constructed only on the basis of radial-basis functions.
\end{abstract}

\section{Introduction}

The most important requirement for finite-element (FE) models of structures is their maximum possible correspondence to real objects by parameters of stress-strain state (SSS) and dynamic characteristics. However, it is impossible or difficult to achieve the required level of coincidence of the numerical solution by the finite element method (FEM) [1] and the results of the full-scale test of the structure under study.

The discrepancy between the results can be minimized by eliminating FE modeling errors, for example, in studying the accuracy-convergence of a numerical solution, as well as aspects of field trials. However, often in this case the required coincidence of the model and the object is not achieved. As a rule, the main reason for this discrepancy are errors in the parameters of the FE models, ignorance of their real values. Such parameters can be geometric characteristics of sections, for example, their areas, moments of inertia. Ignorance of their true values is associated with a variation in the geometric characteristics of the structure due to the presence of cracks, hidden defects. Also, due to the heterogeneity of materials, their mechanical characteristics (moduli of elasticity, coefficients of transverse

* Corresponding author: zav1692@ mail.ru 
deformation, density) cannot be determined at the object of investigation with a sufficient degree of accuracy. For example, the class of concrete construction can vary depending on the conditions of laying concrete, a set of its strength. In this case, in order to minimize the level of discrepancy between the FE model and the real object, it is necessary to solve the problem of refinement (identification) of model parameters [2, 3].

To refine the parameters of FE models, in recent years, methods of responses approximation (RA) have been used. The responses in this paper are characteristics that can be calculated with the help of FEM and measured on a structure using sensors. For example, the responses in the form of static SSS parameters are the displacement, deformation, and stresses of the deformed solid from various static loading options. Dynamic characteristics can also be used in the form of natural frequencies of vibration of structures.

The RA methods are used with a much smaller resource of the computer than by other methods of FE models (sensitivity analysis methods [4, 5], stochastic [6, 7], optimization [8, 9]). This is explained by the fact that all actions to find the optimal parameter values (directly to refine them) are performed on the approximation models of responses. It should be noted that the RA methods are relatively simple in software implementation and do not require any of their integration with FE solvers.

The RA models depend on the parameters to be refined and are built for a fixed number of iterations (launching the FE model for calculation) with the known specified values of the refined parameters to obtain the required responses of the FE model. The rules for determining the number of launches of the FE model for calculation and the values of the parameters for these launches are called experiment plans, or simply plans, but directly the vector of refined parameters on the iteration underway - the starting point. The responses that are computed on the FE model with parameter values corresponding to the origin points are called initial responses.

Polynomial regression models are usually used as RA models [10], which stand out against the backdrop of many other models by the simplicity and intuitive nature of their construction, and also by their clarity. However, the accuracy of such models is low and for their construction requires a large number of initial points - the launch of the FE model for calculation. At the same time, the number of these launches increases in a geometric progression with an increase in the number of refined parameters.

To overcome the disadvantages of using polynomial regression models, it is necessary to use more accurate RA models, which are generated on as few initial points as possible. These RA models are the models based on radial-basis functions [11] and Kriging models $[12,13]$, which are models of spatial interpolation and are based on constructing the correlation structure of data obtained from the FE model.

Thus, the goal of this paper is to compare the efficiency of approximation of responses by models based on radial-basis functions and Kriging models. The work is carried out using the example of a numerical experiment to refine the parameters of the FE model of a simple beam. Its parameters vary in length. To achieve the goal of the work, it is necessary to estimate the reliability of the description of these models of the dependence of the FE model responses on the parameters being refined, as well as the solution of the problem of searching for a vector of optimal parameter values for comparing the speed of computation of responses on these models.

\section{Basic mathematical dependencies}

The RA models, in the present paper, are constructed on special plans in the form of Latin squares (LS) [14], the main feature of which is the uniform filling of the parameter area by the minimum possible number of initial points. The parameter area is determined by the known boundary values of the parameters. 
In one of the LS variants, these are plans for which a certain coordinate on parameters appears only once in a row and only once in the matrix column of the plan [X]. The number of rows of the matrix $[X]$ is the minimum required number of initial points of the Latin squares $N_{t}$, which depends on the number $k$ of parameters to be refined. The number of columns of the matrix $[X]$ is equal to the number of parameters to be refined. The number of points $N_{t}$ is calculated according to formula:

$$
N_{t}=0.5 \cdot(k+1) \cdot(k+2) .
$$

With the FE models, in most cases, more than one response is approximated, and for each of them, a separate RA model is constructed. For this, after the LS formation, at each point of the plan, the necessary initial responses are calculated, as well as their $n$ vectors $\left\{Y_{f e m}\right\}, j=1, \ldots, n$. These vectors, together with the plan matrix $[X]$, are the initial data in the construction of the RA models.

The basic idea of RA models being constructed on the basis of radial-basis functions $(\mathrm{RBF})$ is to construct a matrix of radial-basis functions $[\Phi]$ and to evaluate the influence of each point of the plan on the whole range of parameters from the responses $\left\{Y_{\text {fem }}\right\}_{j}$. The matrix $[\Phi]$ is a square matrix whose dimension is determined by the number of points in the plan:

$$
[\Phi]=\left(\begin{array}{ccc}
\varphi\left(\left|x_{1}-x_{1}\right|\right) & \cdots & \varphi\left(\left|x_{1}-x_{N t}\right|\right) \\
\vdots & \ddots & \vdots \\
\varphi\left(\left|x_{N t}-x_{1}\right|\right) & \cdots & \varphi\left(\left|x_{N t}-x_{N t}\right|\right)
\end{array}\right) .
$$

Each element of this matrix is a function $\varphi(r)$, depending on the distance $r$ between the initial points $x_{i}$ and $x_{l}, i, 1=1, \ldots, N_{t}$. In the present paper, as a radial-basis function, a multiquadratic equation of the form:

$$
\varphi(r)=\left(r^{2}+\sigma^{2}\right)^{0.5}
$$

where $\sigma$ is the coefficient that is selected before a high level of correspondence of the RBF model responses to the responses calculated at the special control points.

After constructing the matrix $[\Phi]$, it is necessary for each RBF model to calculate the weight vector $\{W\}_{j}, j=1, \ldots, n$, which determines the "weight" of each point in the parameter area, depending on the input data in the form $[X]$ and $\left\{Y_{f e m}\right\}_{j}$. This vector is calculated under the assumption that the responses of the RA models at the initial points are equal to the initial responses:

$$
\{W\}_{j}=[\Phi]^{-1} \cdot\left\{Y_{\text {fem }}\right\}_{j} .
$$

After computing $\{W\} j$, the $j$-th RBF model of RA has the form:

$$
y_{j}=[\Phi] \cdot\{W\}_{j} .
$$

Kriging-models are similar to RBF-models in terms of the preliminary construction of a square matrix of distance functions between the points of the plan. In Krieging models, such a matrix is called a correlation matrix and is denoted as $[R] j, j=1, \ldots, n$, and it is unique for each RA model. Its dimension is determined by the number of points of the LS, and it has the form: 


$$
\begin{gathered}
{[R]_{j}=\left(\begin{array}{ccc}
r\left(x_{1}, x_{1},\{\theta\}_{j}\right) & \cdots & r\left(x_{1}, x_{N t},\{\theta\}_{j}\right) \\
\vdots & \ddots & \vdots \\
r\left(x_{N t}, x_{1},\{\theta\}_{j}\right) & \cdots & r\left(x_{N t}, x_{N t},\{\theta\}_{j}\right)
\end{array}\right),} \\
r\left(x_{i}, x_{l},\{\theta\}_{j}\right)=\exp \left(-\sum_{s=1}^{k}\{\theta\}_{j_{s}}\left(\left\{x_{i}\right\}_{s}-\left\{x_{l}\right\}_{s}\right)^{2}\right),
\end{gathered}
$$

where $r\left(x_{i}, x_{l},\{\theta\}_{j}\right)$ is the correlation function, $\left\{x_{i}\right\},\left\{x_{l}\right\}$ are the vectors of coordinates of initial points with indices $i$ and $l ;\{\theta\} j$ is a vector of weight coefficients with respect to parameters; $s$ is the parameter index.

To generate $n$ of Kriging models, the optimization problem, individual for each RA model, is solved, which consists in maximizing the likelihood function $\ln (L)$ by varying the values of the vector $\{\theta\} j, j=1, \ldots, n$ :

$$
\ln (L)=-\frac{\left(N_{t} \cdot \ln \left(\sigma_{j}^{2}\right)+\ln \left(\operatorname{det}[R]_{j}\right)\right)}{2},
$$

where $\operatorname{det}[R]_{j}$ is the determinant of the matrix $[R]_{j} ; \sigma_{j}^{2}$ is the variance estimate of the approximated response.

To calculate the value of the $j$-th response $y_{w j}$ with the help of its Kriging model at the new measurement point $x_{w}$, it is necessary to create a correlation vector between the initial and complementary measurement points $\{r\}_{w j}$ of the following form:

$$
\{r\}_{w j}=\left(\begin{array}{c}
r\left(x_{1}, x_{w},\{\theta\}_{j}\right) \\
\vdots \\
r\left(x_{N t}, x_{w},\{\theta\}_{j}\right)
\end{array}\right),
$$

where $r_{i w}$ is the correlation function between the starting point $x_{i}$ and the new measurement point $x_{w} . r_{i w}$ is calculated by the formula (7).

Direct calculation of the value of the $j$-th response $y_{w j}$ on the new measuring point $x_{w}$ with the help of the Kriging model is carried out by a formula of the form:

$$
y_{w j}=\mu_{j}+\{r\}_{w j}^{T} \cdot[R]_{j}^{-1} \cdot\left(\left\{Y_{\text {fem }}\right\}_{j}-\mu_{j}\{E\}\right)
$$

where $\{E\}$ is a vector of dimension $N t \times 1$, consisting of ones; $\mu j$ is the estimate of the mathematical expectation of the approximated response.

Reliability of the description of the dependence of the response on the refined parameters by the RA model is estimated using the determination coefficient $R^{2}(11)$ and checked at the control points being generated automatically. This coefficient is calculated for each RA model separately, and it shows the fraction of the response variance explained by the approximation model in its total variance. If $R^{2}>0.9$, then the model reflects the approximated dependence with a sufficient degree of accuracy. The maximum possible value of the coefficient is $1 \mathrm{a}$.

$$
R^{2}=1-\frac{\sum_{i=1}^{T}\left(y_{c i}-y_{i}\right)^{2}}{\sum_{i=1}^{T}\left(y_{c i}-y_{c a v}\right)^{2}},
$$


where $y_{c i}$ is the response computed by the FE model at the reference point with the index $i$; $y_{c a v}$ - the average response value from all control points; $y_{\mathrm{i}}$ is the response computed on the RA model at the point with coordinates corresponding to the reference point with the index $i$.

As a method of optimization, a heuristic genetic method is used in the work. Genetic optimization methods [15] are based on modeling the process of natural selection within a "population" consisting of "individuals." Each individual has its own "genes" or the vector of values of the parameters $\{X\}$ and its value of the objective function $F_{o b j}(\{\delta\})$. The Euclidean norm of deviation $\{\delta\}$ responses $\{Y\}$, calculated on the models of their approximation from the responses fixed on the $\left\{Y_{T E S T}\right\}$ construction with allowance for the maximum modulus deviation, is used as the objective function in the work. Thus, the objective function has the form:

$$
\begin{gathered}
F_{o b j}(\{\delta\})=\sqrt{\sum_{j=1}^{n}\left(\{\delta\}_{j}\right)^{2}}+\max \left(\left|\{\delta\}_{j}\right|\right), \\
\{\delta\}=\{Y\}-\left\{Y_{\text {TEST }}\right\} .
\end{gathered}
$$

\section{Refinement of the parameters of the finite element beam model}

To compare the efficiency of approximation of the RBF and Kriging-model responses, a numerical experiment was performed to refine the parameters of the FE model of a beam 20 $\mathrm{m}$ long with a box section shown in Fig. 1 .

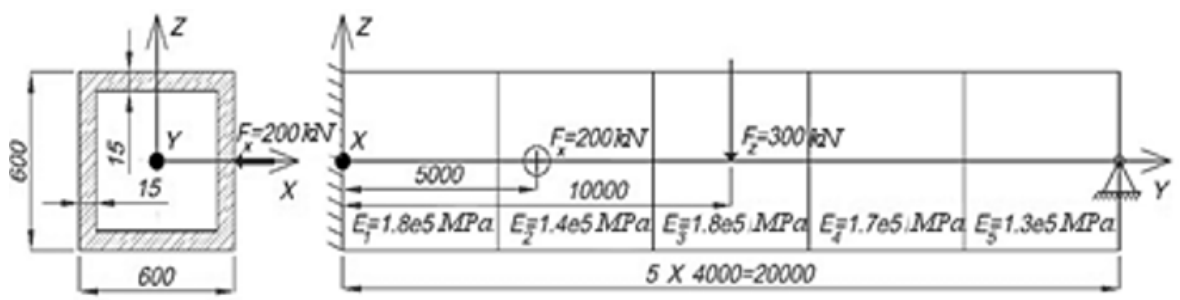

Fig. 1. The dimensions of the beam ( $\mathrm{mm}$ ), the shape of its section, and the acting loads.

The FE model (FE-P) of a beam, with the known correct values of the parameters in the form of elastic moduli, from which the responses of the "natural tests" $\left\{Y_{T E S T}\right\}$ are extracted, is divided into 5 groups of finite elements with a length of $4 \mathrm{~m}$. Each of them is given a unique modulus of elasticity with the complete identity of the remaining characteristics (Poisson's ratio $v=0.28$, density $p=7800 \mathrm{~kg} / \mathrm{m}^{3}$ ). The numbers of the FE groups and their elastic moduli are presented in Table 1 and Fig. 1. The intervals of variation of the elastic moduli of all FE groups ([123500, 256500] MPa) form a range of parameters. These intervals are normalized in such a way that the minimum value of the parameter corresponds to -1 , and the maximum is +1 .

The responses that make up $\left\{Y_{\text {TEST }}\right\}$ are presented in Table 2 in the form:

1. Moving the central point of the beam in the vertical direction $Z_{m}$ when applying a vertical load $F_{z}$ to this point;

2. Moving a point corresponding to a quarter of the length of the beam in the direction of the x-axis - $X_{q}$, when applying a horizontal load $F_{x}$ to this point;

3 . The first 3 eigenfrequencies of the beam oscillations $v_{1}-v_{3}$. 
Table 1. Values of the parameters: correct; initial when being optimized; refined.

\begin{tabular}{|c|c|c|c|c|c|c|c|}
\hline No & $\begin{array}{l}\text { Parameter } \\
\text { (moduli of } \\
\text { elasticity) }\end{array}$ & $\begin{array}{c}\text { True } \\
\text { values } \\
\text { of } \mathrm{E}_{\mathrm{r}} \text {, } \\
10^{4} \mathrm{MPa}\end{array}$ & $\begin{array}{l}E_{0} \\
\text { dev- } \\
\text { on } \\
\text { from } \\
E_{r}, \%\end{array}$ & $\begin{array}{c}\text { Refining } \\
\text { values of } E_{\mathrm{u}} \\
\text { parameters } \\
\text { in RBF } \\
\text { models, } \\
\text { MPa }\end{array}$ & $\begin{array}{c}\mathrm{E}_{\mathrm{u}}, \mathrm{dev}-\mathrm{ons} \\
\text { calc-ed in } \\
\mathrm{RBF} \\
\text { models, out } \\
\text { of } \mathrm{E}_{\mathrm{r}}, \%\end{array}$ & $\begin{array}{c}\text { Refining } \\
\text { values of } \\
E_{u} \\
\text { parameters } \\
\text { in Kriging } \\
\text { models, } \\
\text { MPa }\end{array}$ & $\begin{array}{c}E_{u} \text { dev-ons } \\
\text { calc-ed in } \\
\text { Kriging } \\
\text { models, } \\
\text { out of } E_{r} \text {, } \\
\%\end{array}$ \\
\hline 1 & $E_{1}$ & 18 & $-5,56$ & 185280 & 2.9 & 183950 & 2.2 \\
\hline 2 & $E_{2}$ & 14 & $-35,71$ & 138000 & 1.43 & 138560 & 1 \\
\hline 3 & $\mathrm{E}_{3}$ & 18 & $-5,56$ & 178960 & 0.58 & 180290 & 0.16 \\
\hline 4 & $\mathrm{E}_{4}$ & 17 & $-11,76$ & 167260 & 1.61 & 166330 & -2.16 \\
\hline 5 & $E_{5}$ & 13 & $-46,15$ & 129350 & 0.9 & 130150 & 0.12 \\
\hline
\end{tabular}

Table 2. Values of responses at some source points and responses obtained from the FE model with known correct parameters.

\begin{tabular}{|c|c|c|c|c|c|c|c|c|c|c|c|}
\hline \multirow{2}{*}{ No } & \multirow{2}{*}{$\begin{array}{c}\text { Point } \\
\text { numbe } \\
\text { r }\end{array}$} & \multicolumn{5}{|c|}{ Normalized parameter values } & \multicolumn{5}{|c|}{$\begin{array}{l}\text { The response values from the FE model, } \\
\left.\qquad\left\{Y_{F E M}\right\}_{j} \text { (line } 4-\left\{Y_{T E S T}\right\}\right)\end{array}$} \\
\hline & & $\mathbf{X}_{1}$ & $\mathbf{X}_{2}$ & $\mathbf{X}_{3}$ & $\mathbf{X}_{4}$ & $\mathbf{X}_{5}$ & $\begin{array}{l}\mathbf{Z}_{\mathrm{m}} \\
\mathbf{M M}\end{array}$ & $\mathbf{X}_{\mathbf{q}}, \mathbf{M M}$ & $\mathbf{v}_{1}, \mathbf{\Gamma ц}$ & $\mathbf{v}_{2}, \mathbf{\Gamma ц}$ & $\begin{array}{l}\mathbf{v}_{3}, \\
\text { ГЦ }\end{array}$ \\
\hline 1 & 1 & +0.3 & +0.1 & +0.4 & +0.6 & -1.0 & -57. & -10.7 & 7.2 & 9.8 & 22.1 \\
\hline 2 & 10 & +1.0 & -0.3 & +0.5 & -0.5 & +0.1 & -52.63 & -10.2 & 6.7 & 9.5 & 21.9 \\
\hline 3 & 21 & -0.4 & +0.9 & -0.1 & +0.4 & +0.2 & -60.5 & -9.9 & 7.1 & 10.3 & 23.7 \\
\hline 4 & \multicolumn{6}{|c|}{$Y_{\text {TEST }}$} & -64.6 & -11.6 & 6.8 & 9.4 & 20.6 \\
\hline
\end{tabular}

The task of the numerical experiment is to evaluate the efficiency of the RA models, which are built on the basis of data from the corrected FE model (FE-K), based on such criteria as the reliability of the description of the response dependencies on the parameters and the computational speed on the RA models when solving the optimization problem.

The FE-K differs from the FE-P by equal moduli of elasticity of groups of finite elements; particularly, they are equal $\mathrm{E}_{0}=1,9 \cdot 10^{5} \mathrm{MPa}$.

The RA models are built on a LS with the 21 st reference point, i.e. to obtain models of RA, FE-K is launched for calculation 21 times with the values of the parameters of the LS plan. Some of the points' coordinates in the plan, along with the initial responses, are presented in Table 2. When constructing RBF models, weights are calculated by the formula (4), and for the Kriging models, based on the results of solving the optimization problem (8), the weighting coefficient vector by parameters.

Verification of RA models is to determine the coefficients of their determination. To do this, 500 measurement points are randomly generated in the parameter area. It is worth noting that such a number of points is chosen for complete verification of the RA models and when solving real-world problems, the FE models need only generate 10-20\% of control points relative to the number of points in the plan. The values of the determination coefficients are presented in Table 3, and they are all close to 1e.

Table 3. Coefficients of determining response approximation models.

\begin{tabular}{|c|c|c|c|c|c|c|}
\hline \multirow{2}{*}{ No } & $\begin{array}{c}\text { Coefficient of } \\
\text { determination of } \\
\text { models }\end{array}$ & $\mathbf{Z}_{\mathbf{m}}$ & $\mathbf{Z}_{\mathbf{q}}$ & $\mathbf{v}_{\mathbf{1}}$ & $\mathbf{v}_{\mathbf{2}}$ & $\mathbf{v}^{\mathbf{n}}$ \\
\cline { 3 - 7 } & RBF models & 0,986 & 0,993 & 0,996 & 0,998 & 0,999 \\
\hline 1 & Kriging models & 0,998 & 0,996 & 0,997 & 0,995 & 0,998 \\
\hline 2 &
\end{tabular}

To solve the problem of searching for the parameters of the FE-K, a target function of the form (12) is formed. Further, the difference between the responses of the "natural tests" $\left\{Y_{T E S T}\right\}$ obtained on the FE-P model and the responses of the RA models based on the FE$\mathrm{K}$ is minimized. Varying parameters in the solution of the optimization problem are the 
refined parameters of the RA models. To determine the average value of the objective functions and elastic moduli of each finite element group, the genetic optimization algorithm was launched 50 times. The results of these calculations are presented in Table 4. The results of calculations with the minimum (best) identified objective functions are presented in Table 1.

Table 4. Results of solving the optimization problem.

\begin{tabular}{|c|c|c|c|}
\hline No & $\begin{array}{c}\text { Model } \\
\text { type }\end{array}$ & Index & Value \\
\hline 1 & \multirow{2}{*}{$\begin{array}{c}\text { RBF } \\
\text { models }\end{array}$} & Average time to achieve convergence, sec. & 76,4 \\
\hline 2 & & Average value of the objective function & 0,34 \\
\hline 3 & \multirow{2}{*}{$\begin{array}{l}\text { Kriging } \\
\text { models }\end{array}$} & Average time to achieve convergence, sec. & 253,8 \\
\hline 4 & & Average value of the objective function & 0,38 \\
\hline
\end{tabular}

To construct the RA models, verify them, and solve the optimization problem, the programming language Python was used.

\section{Conclusion}

Based on the results of verifying the RA models and solving the optimization problem, the following conclusions can be drawn:

1. RBF- and Kriging-models approximate the dependence of responses on parameters with the same high degree of accuracy when solving the problems of refining the FE models' parameters by the method of approximation of responses. This circumstance makes it possible to use RBF models instead of Kriging models in solving problems of the FE models.

2. The speed of solving the optimization problem for RBF-models is significantly higher in comparison with the Kriging-models, for which additional time is also required. By analogy with point 1 , this indicates the priority of applying the RBF models for the FE models.

3. The genetic method of optimization allows us to study the range of parameters with a great degree of detail for the global minimum of the studied dependences.

4. It is necessary to verify the use of RBF and Kriging RA models on more complex FE models of real objects with a large number of parameters and responses to confirm the results presented in the work.

\section{References}

1. O. S. Zenkevich, Finite Element Method in Engineering (Izd-vo Mir, Moscow, 1975)

2. G. Chen, FE model validation for structural dynamics (University of London, 2001)

3. A. V. Zabelin, A. A. Pykhalov, Vestnik PNIPU - Mechanics, 25(7) (2017)

4. J. E. Mottershead, M. Link, M.I. Friswell, Mechanical Systems and Signal Processing, 25(7) (2011)

5. M. I. Friswell, J. E. T. Penny, Mechanical Systems and Signal Processing, 4(5) (1990)

6. J. R. Fonseca, M. I. Friswell, J. E. Mottershead, A.W. Lees, Journal of Sound and Vibration, 288(3) (2005)

7. H. H. Khodaparast, J. E. Mottershead, K. J. Badcock, Mechanical Systems and Signal Processing, 25(4) (2011)

8. A. Teughels, G.D. Roeck, Computational Methods in Engineering, 12(2) (2005) 
9. Y. X. Zhang, B. F. Spencer, S. H. Sim, SMSST (2007)

10. A. V. Zabelin, A. A. Pykhalov, Transport infrastructure of the Siberian region: proceedings of the Eighth International Scientific and Practical Conference, 2 (2017)

11. M. Mongillo, SIAM Undergraduate Research Online, 4 (2011)

12. S.-S. Jin, H.-J. Jung, Computers \& structures, 168 (2016)

13. K. Sun, Y. Zhao, H. Hu, Shock and vibration (2015)

14. A. D. Keedwell, J. Dénes, Latin Squares and their Applications (Second Edition) (North Holland, 2015)

15. D. E. Goldberg, Genetic algorithms in search, optimization and machine learning (Addison-Wesley, 1989) 